\title{
ELECCIONES NACIONALES 2010 EN COSTA RICA: UNA MUJER AL MANDO SESENTA AÑOS DESPUÉS
}

\section{NATIONAL ELECTIONS IN COSTA RICA: A WOMAN PRESIDENT SIXTY YEARS LATER}

\author{
Ronald Alfaro Redondo*
}

\section{RESUMEN}

Las elecciones generales de 2010 no modificaron radicalmente el sistema de partidos en la época post-bipartidista. Dos son los factores fundamentales que caracterizan a este periodo. Por un lado, una composición pluralista y sin mayorías de los órganos de representación política producto de una elevada fragmentación del sistema de partidos $y$ por el otro, la alta volatilidad en las preferencias electorales de la ciudadanía, a pesar de una ligera reducción en el 2010. Como hecho relevante, por primera vez en la historia del país, una mujer ocupa el cargo de Presidente de la República.

PALABRAS CLAVE: COSTA RICA * ELECCIONES * PARTIDOS POLÍTICOS * PLURALISMO POLÍTICO * PARTICIPACIÓN POLÍTICA

\section{ABSTRACT}

The Costa Rican electoral contest of 2010 does not alter political party system in a radical way. Instead, two main features still characterize the current post-bipartisan era. First, the plural composition of the Congress and local governments as a result of the high fragmentation that prevails in the party system. Second, in spite of the fact that electoral preferences' (volatility) decreased slightly in 2010, inter-election voting swings remain high. Finally, sixty years after the country adopted universal suffrage a woman selected president.

KEY WORDS: COSTA RICA * ELECTIONS * POLITICAL PARTIES * POLITICAL PLURALISM * POLITICAL PARTICIPATION

Programa Estado de la Nación auspiciado por el Consejo Nacional de Rectores (CONARE) y la Defensoría de los Habitantes de la República. ralfaro@estadonacion.or.cr 


\section{INTRODUCCIÓN}

El 7 de febrero de 2010, los costarricenses efectuaron el décimo quinto proceso electoral consecutivo desde 1953. Producto de esta larga trayectoria, Costa Rica constituye la democracia electoral más estable en la región latinoamericana. En esta oportunidad se renovaron los cargos de presidente $y$ vicepresidentes de la República, diputados a la Asamblea Legislativa y concejales municipales en las 81 municipalidades del país. En total se designaron 1050 cargos en todo el país. El Partido Liberación Nacional - la agrupación más longeva logró mantener bajo su control el Poder Ejecutivo por dos períodos. Además, cuenta con la fracción más numerosa en el
Congreso - aunque sin alcanzar mayoría simple - y la mayor cantidad de regidores en las municipalidades.

Como hecho relevante, por primera vez en la historia del país, una mujer ocupa el cargo de Presidente de la República. Asimismo, nuevas reglas electorales entraron a regir justo antes de la realización de los comicios. En términos generales, las elecciones se desarrollaron en un ambiente de normalidad. Los comicios presidenciales se definieron por un amplio margen de diferencia, no así la elección parlamentaria cuyos resultados arrojaron un Congreso sin mayoría y con representación de ocho fuerzas políticas. Por último, el porcentaje de electores que se abstuvo de participar disminuyó por primera vez desde 1998.

CUADRO 1

RESUMEN DE RESULTADOS DE LA ELECCIÓN GENERAL 2010

\begin{tabular}{lccccc}
\hline PARTIDO POLÍTICO & \multicolumn{2}{c}{ ELECCIÓN PRESIDENCIAL } & \multicolumn{2}{c}{ ELECCIÓN LEGISLATIVA } & \multirow{2}{*}{ ESCAÑOS } \\
\cline { 2 - 5 } & ABSOLUTOS & $\%$ & ABSOLUTOS & $\%$ & \\
\hline Liberación Nacional & 896516 & 46,9 & 708043 & 37,3 & 24 \\
Acción Ciudadana & 478877 & 25,1 & 334636 & 17,6 & 11 \\
Movimiento Libertario & 399778 & 20,9 & 275518 & 14,5 & 9 \\
Unidad Social Cristiana & 74114 & 3,9 & 155047 & 8,2 & 6 \\
Otros partidos & 62038 & 3,2 & 426581 & 22,5 & 7 \\
\hline TOTAL VOTOS VÁLIDOS & 1911333 & 100,0 & 1899825 & 100,0 & 57 \\
\hline
\end{tabular}

* La categoría "otros partidos" incluye en la elección presidencial a los restantes 5 partidos políticos, 4 de los cuales no alcanzaron siquiera el $1 \%$ de los votos válidos emitidos. En el caso de la elección legislativa, dicha categoría agrupa a los restantes 14 partidos políticos, 1 de los cuales obtiene 4 diputados y 3 de los cuales obtienen 1 diputado.

Fuente: elaboración propia con información del TSE.

\section{REGLAS ELECTORALES}

En las elecciones generales de 2010 se aplicaron nuevas reglas electorales producto de la aprobación de un nuevo Código Electoral en 2009. La legislación vigente reformó tres áreas medulares en este tema: fortalecimiento de la institucionalidad electoral, ampliación de derechos civiles y políticos, y fortalecimiento de los partidos políticos. En cuanto a la primera de estas áreas, sobresalen dos nuevas instancias internas al Tribunal Supremo de Elecciones (TSE), la primera lo constituye un nuevo organismo electoral denominado Registro Electoral, cuyas responsabilidades principales son administrar el registro de partidos políticos y controlar las contribuciones privadas $y$ del Estado a los partidos políticos. La segunda entidad creada es una dependencia interna llamada Instituto de Formación y Estudios para la Democracia (IFED) y fue creada con la misión de diseñar y ejecutar programas de formación para promover una 
ciudadanía activa y capacitación para los partidos políticos.

Sobre la expansión de derechos civiles y políticos, el nuevo Código garantiza el derecho al sufragio de los ciudadanos costarricenses que residen en el exterior, quienes podrán votar en las elecciones presidenciales de 2014. Asimismo, se establece la paridad de género en la conformación de papeletas y órganos internos de los partidos políticos.
Finalmente, una de las áreas de la normativa que mayores cambios experimentó fue la de financiamiento político. En la última década se dieron a conocer irregularidades en la administración de las finanzas públicas y privadas de los partidos políticos, algunas de las cuales incluso llegaron a instancias judiciales. En la Tabla 1 se resumen algunas de las principales novedades de la nueva legislación electoral.

TABLA 1

CARACTERÍSTICAS DE LA NUEVA LEGISLACIÓN ELECTORAL COSTARRICENSE

\begin{tabular}{|c|c|}
\hline COMPONENTES & CARACTERÍSTICAS \\
\hline $\begin{array}{l}\text { Institucionalidad } \\
\text { electoral }\end{array}$ & $\begin{array}{l}\text { Creación del Registro Electoral y del Instituto de Formación y Estudios para la Democracia (IFED). } \\
\text { Se le da rango legal a la jurisprudencia electoral. }\end{array}$ \\
\hline $\begin{array}{l}\text { Derechos civiles y } \\
\text { políticos }\end{array}$ & $\begin{array}{l}\text { Paridad de género en la conformación de las papeletas en los distintos tipos de elecciones y órganos } \\
\text { internos de los partidos políticos. } \\
\text { Instauración del voto en el extranjero. }\end{array}$ \\
\hline $\begin{array}{l}\text { Financiamiento } \\
\text { electoral }\end{array}$ & $\begin{array}{l}\text { Dota de financiamiento público a las elecciones municipales. } \\
\text { Mayores controles al financiamiento público y privado de los partidos políticos. } \\
\text { Se prohíbe la donación de personas jurídicas a la campaña. } \\
\text { Cuenta bancaria única para administrar las donaciones de campaña. } \\
\text { Las finanzas de los partidos podrán ser auditadas. } \\
\text { Se crean la figura del delito electoral y se establecen sanciones. }\end{array}$ \\
\hline
\end{tabular}

Fuente: elaboración propia con base en el Código Electoral.

A diferencia de otros sistemas electorales, en Costa Rica no es necesario inscribirse para sufragar, pues el registro de electores es automático. Un total de 2822491 electores estaban habilitados para votar de acuerdo con el Registro Civil. Esta cifra representó un 10,7\% más que los registrados en el 2006. El $76 \%$ de los electores residía en las provincias (o departamentos) centrales y el $24 \%$ restante pertenecía a las regiones periféricas (fronteras y costas: Guanacaste, Puntarenas y Limón). En esta oportunidad, el TSE habilitó 6617 urnas electorales en todo el país en 2040 centros de votación. A partir de la elección de 1998, el TSE adoptó la decisión de disminuir el número de juntas receptoras de votos aumentando el promedio de electores por recinto. Como resultado, en las elecciones de 2010, el número de juntas receptoras fue similar al de
1986, cuando el padrón electoral era la mitad del actual.

Las elecciones se desarrollaron en un ambiente de normalidad $y$ no se reportaron denuncias por coacción, razón por la cual dichos comicios pueden ser considerados como libres. De esta forma, una vez más, el resultado electoral reflejó la voluntad popular. El amplio margen de diferencia entre Laura Chinchilla (PLN) y Ottón Solís (PAC), 22 puntos porcentuales, es el tercero más alto de los últimos 57 años, únicamente superada por los 30 puntos porcentuales de diferencia en 1953, entre José Figueres Ferrer y Fernando Castro Cervantes y los 25 puntos porcentuales de diferencia entre Luis Alberto Monge y Rafael Ángel Calderón, en 1982.

A diferencia de las elecciones presidenciales del 2006 y a raíz del amplio margen de diferencia entre el primer $y$ el segundo partido 
político más votado, en esta ocasión los partidos no presentaron una cifra elevada de demandas de nulidad. En aquella oportunidad el Partido Acción Ciudadana (PAC) formuló ante el TSE 681 de las 696 demandas de nulidad presentadas. En dichos recursos la agrupación adujo principalmente extravío de papeletas sobrantes, faltantes de padrones de registro de la votación y ausencia del acta con el registro de los votos. El órgano electoral rechazó todas las demandas formuladas y reafirmó con ello que a pesar de las inconsistencias registradas, el resultado reflejó la voluntad popular (Programa Estado de la Nación, 2007). Producto de los acontecimientos del 2006 mencionados y del referéndum sobre el Tratado de Libre Comercio en 2007, se incrementó significativamente el escrutinio ciudadano sobre las actuaciones de la organización electoral y sus posibles efectos, así como, la vulnerabilidad de la que puede ser objeto un proceso, cuyo uno de sus pilares fundamentales es la integración voluntaria de las juntas receptoras de votación.

En 2010, se repitió la desidia de un grupo de miembros de mesa quienes, a pesar de haber sido designados por los partidos políticos, desistieron de dicho nombramiento y provocaron que algunas mesas receptoras de votos se conformaran con uno o dos miembros. La diferencia con 2006 es que el TSE preparó esta vez, un plan de contingencia basado en el reclutamiento de auxiliares electorales preparados para ocupar el espacio de los miembros propietarios ausentes. Esta situación impidió que las ausencias dieran al traste con la administración de la junta receptora de votos, pero de acuerdo con la nueva legislación, el nombramiento de dichos miembros sigue recayendo exclusivamente en los partidos políticos.

\section{MERMA INESPERADA EN EL ABSTENCIONISMO}

Un elemento novedoso de las elecciones generales 2010, hasta cierto punto inesperado, es el incremento de la participación electoral en 4 puntos porcentuales con respecto a lo acontecido en 2006. Con ello, el porcentaje de electores que no ejercieron el derecho al voto se sitúa en niveles similares a los reportados en 1998. Contra muchos pronósticos, una campaña que daba la impresión de no haber atraído a amplios sectores de la población, resultó ser la elección en la que ejercen el voto el mayor porcentaje de costarricenses, en los últimos 12 años. Con ello se interrumpe una tendencia creciente del abstencionismo de tres elecciones en el periodo 1998-2006.

A pesar de ello, un patrón que no se modifica y permanece invariable desde 1953, es la alta ausencia de electores en las tres provincias periféricas: Guanacaste, Puntarenas y Limón; las cuales han sido y continúan siendo, las provincias en donde menos se ejerce el sufragio. Estas provincias son a su vez, las regiones de menores niveles de desarrollo y menores oportunidades para sus pobladores, lo cual apunta a la existencia de brechas políticas que se manifies$\tan$ en la ausencia de los electores en las urnas.

El Partido Liberación Nacional (PLN) fundado en 1951, de tradición socialdemócrata y hoy día catalogado como centrista, ganó la elección con el $47 \%$ de los votos. En segundo lugar, se ubicó el Partido Acción Ciudadana (PAC, creado en el 2001) de inclinación centroizquierda con un distante 25\%. En tercera posición, se situó el Movimiento Libertario (ML), una agrupación de derecha que participaba por cuarta ocasión con el $21 \%$ de los votos. El Partido Unidad Social Cristiana (PUSC) de centro-derecha alcanzó únicamente el $4 \%$.

Las elecciones de 2010 no cambiaron radicalmente el panorama incierto en cuanto a la recomposición del sistema de partidos en nuestro país. Por el contrario, el impase abierto en 2006 luego del desplome en el respaldo electoral del Partido Unidad Social Cristiana $y$ el posicionamiento del PAC como segunda fuerza política se extiende en el tiempo y por lo tanto, se reafirma con ello la etapa de transición del sistema de partidos hacia un nuevo e indefinido estadio.

\section{PRIMERA MUJER PRESIDENTE}

El PLN, fundado en 1951, ganó la elección presidencial por un amplio margen 
y únicamente perdió uno de los 81 cantones del país. El PAC, por su parte, perdió los 30 cantones $(37 \%)$ en los que se había impuesto cuatro años atrás. El Mapa 1 ilustra con toda claridad la dimensión geográfica de estos cambios en el periodo entre elecciones.
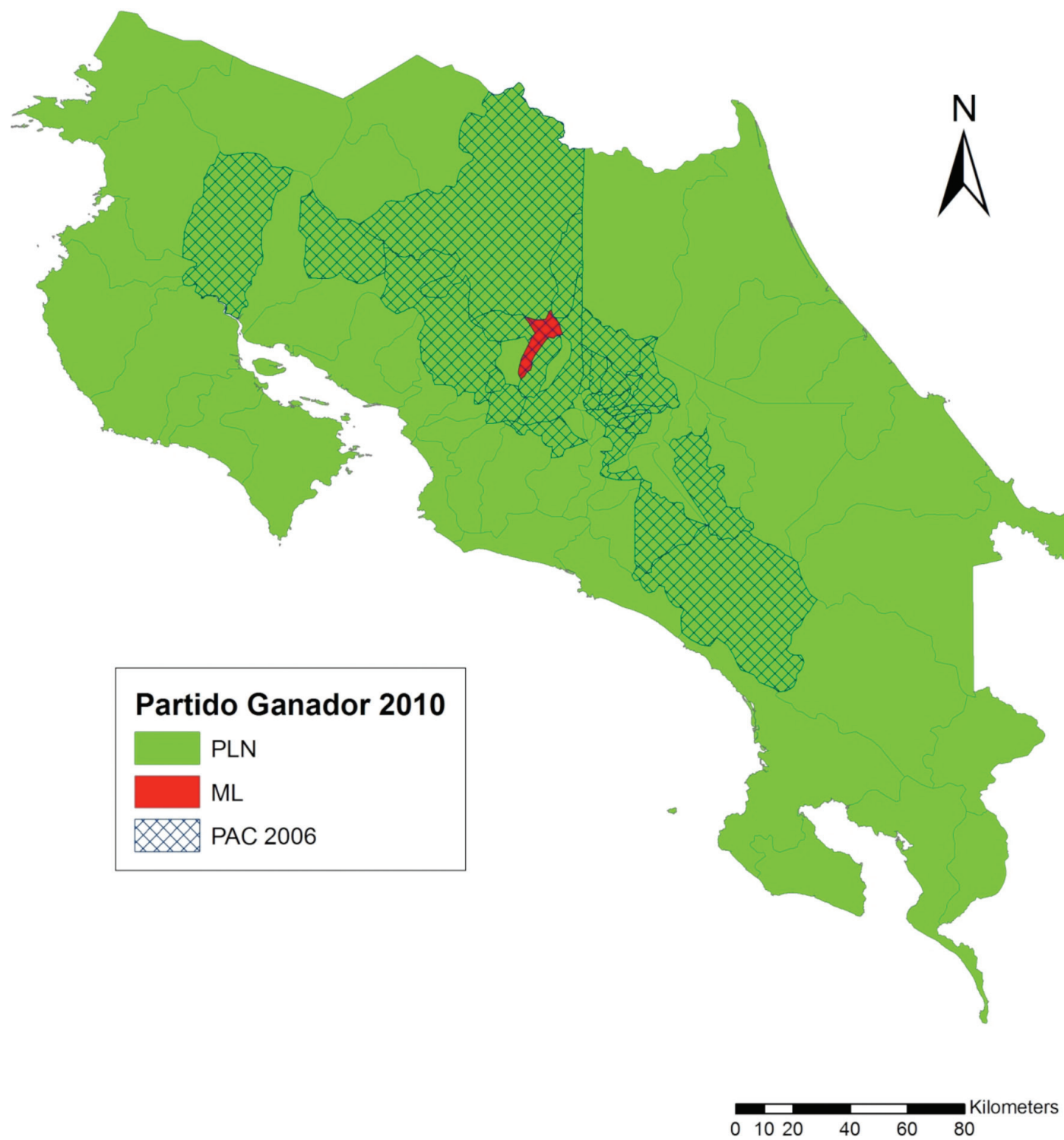

\section{MAPA 1 \\ PARTIDO POLÍTICO GANADOR \\ ELECCIONES PRESIDENCIALES 2006 Y 2010, COSTA RICA}

Fuente: Tribunal Supremo de Elecciones. Elaborado por: Ronald Alfaro Redondo. 
Únicamente en dos elecciones, el liberacionismo ha conseguido ganar en todas las provincias del país: 1953 y 2010. La última vez que este partido logró un triunfo arrasador como el de esta oportunidad fue en 1982, con la elección de Luis Alberto Monge. El PLN logra, 28 años después, de nuevo un triunfo contundente y lleva a la primera ujer a la Presidencia de la República, 60 años después del reconocimiento del derecho al voto a la mujer. Además, 24 años después de dos mandatos consecutivos del PLN (1982-1986 y 1986-1990), esta agrupación tendrá la oportunidad de gobernar durante dos cuatrienios. A pesar de ello, como ya se mencionó, el partido oficialista no contará con mayoría legislativa, tal y como ha sido la regla desde 1994. A diferencia de 2006, en el que el apoyo del PLN provino principalmente, de electores de zonas costeras y fronterizas, caracterizadas por ser mayores extensiones de territorio, con menor densidad poblacional y menores índices de desarrollo humano $y$ de participación política, en esta oportunidad el apoyo del PLN fue homogéneo a lo largo y ancho del país.

El PAC experimentó un descenso en su respaldo electoral, lo que produjo una representación legislativa y municipal considerablemente menor. Aunado al hecho de que el respaldo hacia este partido en la elección presidencial se redujo respecto al 2006, el Partido muestra debilidades para obtener un respaldo electoral geográficamente más homogéneo. Por segunda elección, el PAC recibió el apoyo mayoritariamente de comunidades localizadas en el centro del país, de mayor concentración de población y mayor desarrollo humano. Asimismo, similar a lo acontecido en 2002, el PAC no logró superar lo que constituye su principal obstáculo electoral: obtener mayor cantidad de votos en las provincias costeras $y$ fronterizas a pesar de su fuerte arraigo en el centro del país.

Con los resultados mencionados, el desplazamiento del comportamiento electoral del eje centro-centro/derecha personificado en el PUSC-PLN, hacia un nuevo eje esta vez centro-centro/izquierda con el protagonismo del PLN-PAC, con el que se había caracterizado el panorama electoral derivado de las elecciones de 2006, se matiza en 2010, debido al repunte del Movimiento Libertario que desplaza al PUSC como principal fuerza política de centro/derecha. El ML que en las últimas dos elecciones se ubicó como la tercera fuerza, aglutinó el apoyo del PUSC en las provincias periféricas, regiones en las que esta última agrupación fue tradicionalmente fuerte.

\section{COMICIOS LEGISLATIVOS}

Por otra parte, en lo que respecta a la contienda legislativa, en comparación con la composición parlamentaria del 2006, la fracción de Liberación Nacional disminuyó de 25 a 24 diputados, el PAC de 17 a 11, el Movimiento Libertario obtuvo 9 legisladores, el PUSc incrementó su fracción de 5 a 6 parlamentarios, el Partido Accesibilidad sin Exclusión pasó de 1 a 4 y los restantes 3 partidos que obtuvieron representación legislativa son unipersonales ${ }^{1}$. Por quinta elección consecutiva desde 1994, ninguna agrupación política cuenta con mayoría parlamentaria (29 diputados), lo cual obliga a todas las fuerzas a establecer procesos de negociación para la aprobación de leyes. La Asamblea Legislativa 2010-2014 se caracteriza por ser, junto con las del período 1974-1978 y 2006-2010, las de mayor fragmentación dada la representación de 8 partidos políticos. El antecedente más cercano en esta materia se dio en el período 1998-2002, en el que 7 partidos se distribuyeron los 57 escaños.

La composición histórica del Congreso (1953-2010) por familias de partidos políticos ${ }^{2}$

1 Se trata de los casos del Frente Amplio (FA), Restauración Costarricense (PRC) y Restauración Nacional (PRN).

$2 \quad$ El término familias de partidos políticos en Costa Rica lo empleó el politólogo Fernando Sánchez (2004) para agrupar a la amplia gama de agrupaciones políticas que compiten en los diferentes procesos electorales en tres categorías: 1) el Partido Liberación Nacional (que agrupa a un solo partido, denominada Partido de centro), 2) los partidos de la Alianza Conservadora (llamada Partidos de derecha y centro/derecha) y 3) los partidos de izquierda (Partidos de izquierda $y$ centro/izquierda). En este documento se agrega 
muestra la concentración de los escaños legislativos en los partidos de centro-centro/ derecha. A pesar del repunte de las agrupaciones de izquierda y centro/izquierda en 2006, en 2010 las fuerzas del polo ideológico predominante en la política costarricense recuperaron una proporción importante de sus curules. Esto queda demostrado en el Cuadro 2.

CUADRO 2

COMPOSICIÓN DE LA ASAMBLEA LEGISLATIVA SEGÚN FAMILIA DE PARTIDOS POLÍTICOS 1953-2010

\begin{tabular}{|c|c|c|c|c|c|c|c|c|c|c|c|c|c|c|c|}
\hline \multirow{2}{*}{$\begin{array}{l}\text { a/FAMILIA DE } \\
\text { PARTIDOS POLÍTICOS }\end{array}$} & \multicolumn{15}{|c|}{ AÑOS } \\
\hline & 53 & 58 & 62 & 66 & 70 & 74 & 78 & 82 & 86 & 90 & 94 & 98 & 02 & 06 & 10 \\
\hline Partido de centro & 30 & 20 & 29 & 29 & 32 & 27 & 25 & 33 & 29 & 25 & 28 & 23 & 17 & 25 & 24 \\
\hline $\begin{array}{l}\text { Partidos de derecha y } \\
\text { centro/derecha }\end{array}$ & 15 & 25 & 27 & 28 & 23 & 26 & 27 & 19 & 25 & 29 & 25 & 28 & 25 & 12 & 15 \\
\hline $\begin{array}{l}\text { Partidos de izquierda y } \\
\text { centro/izquierda }\end{array}$ & 0 & 0 & 1 & 0 & 2 & 3 & 4 & 4 & 2 & 1 & 2 & 3 & 14 & 18 & 12 \\
\hline $\begin{array}{l}\text { Partidos regionales e } \\
\text { intereses específicos }\end{array}$ & 0 & 0 & 0 & 0 & 0 & 1 & 1 & 1 & 1 & 2 & 2 & 3 & 1 & 2 & 6 \\
\hline TOTAL & 45 & 45 & 57 & 57 & 57 & 57 & 57 & 57 & 57 & 57 & 57 & 57 & 57 & 57 & 57 \\
\hline
\end{tabular}

a/ Categorías:

1) Partido de centro: se trata del Partido Liberación Nacional, la agrupación política de mayor trayectoria en el país. El PLN fundado en 1951 emergió como una agrupación de inclinación socialdemócrata. Sin embargo, posteriormente modificó su carácter ideológico y actualmente constituye un partido centrista.

2) Partidos de derecha y centro/derecha: incluye a los partidos Demócrata, Republicano Nacional Independiente, Unión Nacional, Republicano, Independiente, Unión Cívico Revolucionaria, Unificación Nacional, Demócrata Cristiano, Nacional Independiente, Renovación Democrática, Coalición Unidad, Movimiento Nacional, Unidad Social Cristiana y Movimiento Libertario.

3) Partidos de izquierda y centro/izquierda: agrupa a los partidos Acción Demócrata Popular, Acción Socialista, Frente Popular Costarricense, Pueblo Unido, Alianza Popular, Fuerza Democrática, Acción Ciudadana y Frente Amplio.

4) Partidos regionales e intereses específicos: Unión Agrícola Cartaginés, Acción Democrática Alajuelense, Unión Generaleña, Agrario Nacional, Renovación Costarricense, Acción Laborista Agrícola, Integración Nacional, Accesibilidad sin Exclusión y Restauración Nacional.

Fuente: Elaboración propia con información del TSE.

\section{ELECCIONES MUNICIPALES}

En lo que respecta a las elecciones municipales, el 2010 es la última elección general en la que se nombran autoridades locales junto con autoridades nacionales, pues a partir de febrero de 2016, se unifican todas las elecciones municipales en una misma fecha, justo dos años después de la elección presidencial y legislativa.

una categoría más a las formuladas por Sánchez dada la especificidad de las agrupaciones que la componen, denominada 4) Partidos regionales $e$ intereses específicos.
En 2010, el Partido Liberación Nacional obtuvo 196 concejales municipales (el $40 \%$ ) de un total de 495 en todo el país. Le sigue el Partido Acción Ciudadana con 99, equivalente al $20 \%$. Por debajo del PLN y el PAC se ubican el Movimiento Libertario y el Partido Unidad Social Cristiana con 75 (15\%) y 53 (11\%) plazas, respectivamente. El Partido Accesibilidad sin Exclusión (PASE) obtuvo 23 regidores (5\%). Luego aparecen otras 40 agrupaciones que obtienen 4 plazas o menos en las municipalidades; 20 partidos políticos cantonales alcanzaron 27 escaños, 5 más que 
en 2006. Dentro de este grupo sobresale el Partido Curridabat Siglo XxI, que obtuvo la mayoría de curules en el cantón. Además, por primera vez, cuatro coaliciones de partidos (Frente Amplio y Alianza Patriótica) obtienen cada una un escaño en los cantones de San Ramón, San Carlos, Alfaro Ruiz y Barva.

La composición político-partidaria de las municipalidades ha variado significativamente en el período 1986-2010. Actualmente, la representación política en el nivel mencionado es de carácter pluripartidista, pues pasó de estar predominantemente dominada por el PUSC-PLN, a concejos municipales fragmentados y sin mayoría. En 1986, en 70 municipalidades, algún partido político contaba con mayoría de miembros en el concejo municipal; en 1990, ese número se reduce a 66. Para las elecciones de 1994, la cifra pasa a ser de 46 y disminuye significativamente en el 2002, a tan sólo 10 cantones (Alfaro, 2002). En el período 2010-2016, el PLN obtuvo la mayoría de los concejales únicamente en 11 municipalidades.

\section{NÚMERO EFECTIVO DE PARTIDOS, VOLATILIDAD $^{3}$ Y NACIONALIZACIÓN PARTIDARIA}

En la literatura especializada sobre partidos políticos predomina la idea de que a mayor fragmentación del sistema político mayor dificultad para construir mayorías y que éstas generen acuerdos. Para medir la fragmentación del sistema de partidos políticos se utiliza el indicador denominado Índice número efectivo de partidos. Básicamente, entre más se acerque el valor del índice a 2 , se está en presencia de un sistema de partidos catalogado como bipartidista y entre mayor el valor, mayor es la fragmentación del sistema. En el caso de Costa

3 Para el cálculo del índice de número efectivo de partidos se utiliza la fórmula $\mathrm{N}=1 / \sum(\mathrm{S})^{2}$, donde (S) es el porcentaje de votos $y / 0$ escaños que obtiene cada partido político. Por su parte, la volatilidad electoral se calcula mediante la fórmula $\mathrm{V}=\left(\sum 1 \% \mathrm{Vt} 1-\% \mathrm{Vt} 01\right) / 2$, en la que $\% \mathrm{Vt1}$ es el porcentaje de votos obtenido por un partido político en la elección t1 (por ejemplo, 2006) y \% Vt0 es el porcentaje de votos de ese mismo partido en la elección t0 (2002).
Rica, el valor del indicador ha sido mayor en la elección presidencial, desde 1978.

En el caso de las elecciones legislativa y municipal, en el 2010, el sistema de partidos costarricense arrojó la mayor fragmentación del sistema político en 57 años. Luego de un período (1986-1998) con un sistema de partidos políticos con presencia predominante de dos agrupaciones (el PUSC y el PLN), las últimas tres elecciones reflejan un incremento de la fragmentación partidaria. Este aspecto se refleja en una composición pluripartidista y sin mayorías del Congreso y las municipalidades, producto de una mayor cantidad de representantes políticos provenientes de distintas agrupaciones (ver gráfico 1).

En lo que a número efectivo de partidos se refiere, a nivel legislativo los valores de 2010 son superiores a los registrados por dicho índice en los procesos electores posteriores a la Guerra Civil de 1948 y los que anteceden a la cristalización del bipartidismo (1970-1978). Mientras que en el caso de las elecciones presidenciales, el índice reporta valores ligeramente inferiores a los de 1974, la elección presidencial de mayor fragmentación desde 1953. A nivel municipal, la tendencia creciente de fragmentación es evidente desde 1990.

El otro aspecto analizado en las elecciones 2006, es la volatilidad electoral, entendida como el desplazamiento del voto entre agrupaciones políticas en dos elecciones consecutivas. Dicho indicador mide la diferencia neta entre las proporciones de votos obtenidos por los partidos políticos en dos elecciones. En 2010 disminuyó la volatilidad electoral especialmente, en las elecciones presidenciales. Vale la pena recordar que este indicador captura los cambios en las preferencias electorales entre partidos políticos. La disminución mencionada debe ser interpretada como un efecto agregado de menor traslado de votos entre agrupaciones políticas, particularmente, en el sentido PLN hacia otros partidos políticos. Los cambios en las preferencias partidarias del 2010 reportados por el índice son ligeramente mayores que los registrados durante el período de vigencia del bipartidismo PLN-PUSC, entre 1986 y 1998, pero no llega a los niveles de los comicios de 1958, 1966 y 1978 (gráfico 2). 


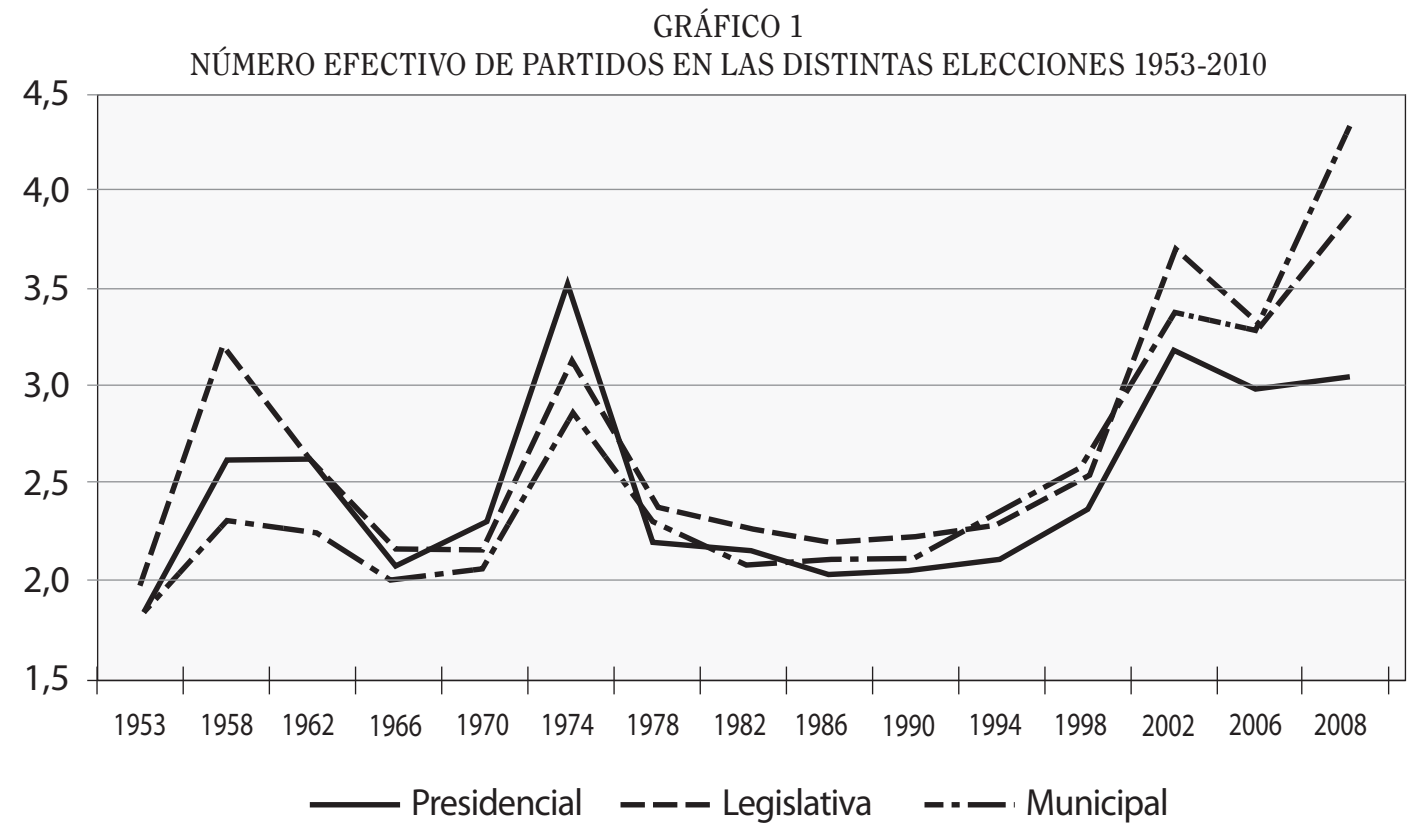

Fuente: Elaboración propia con datos del TSE.

GRÁFICO 2

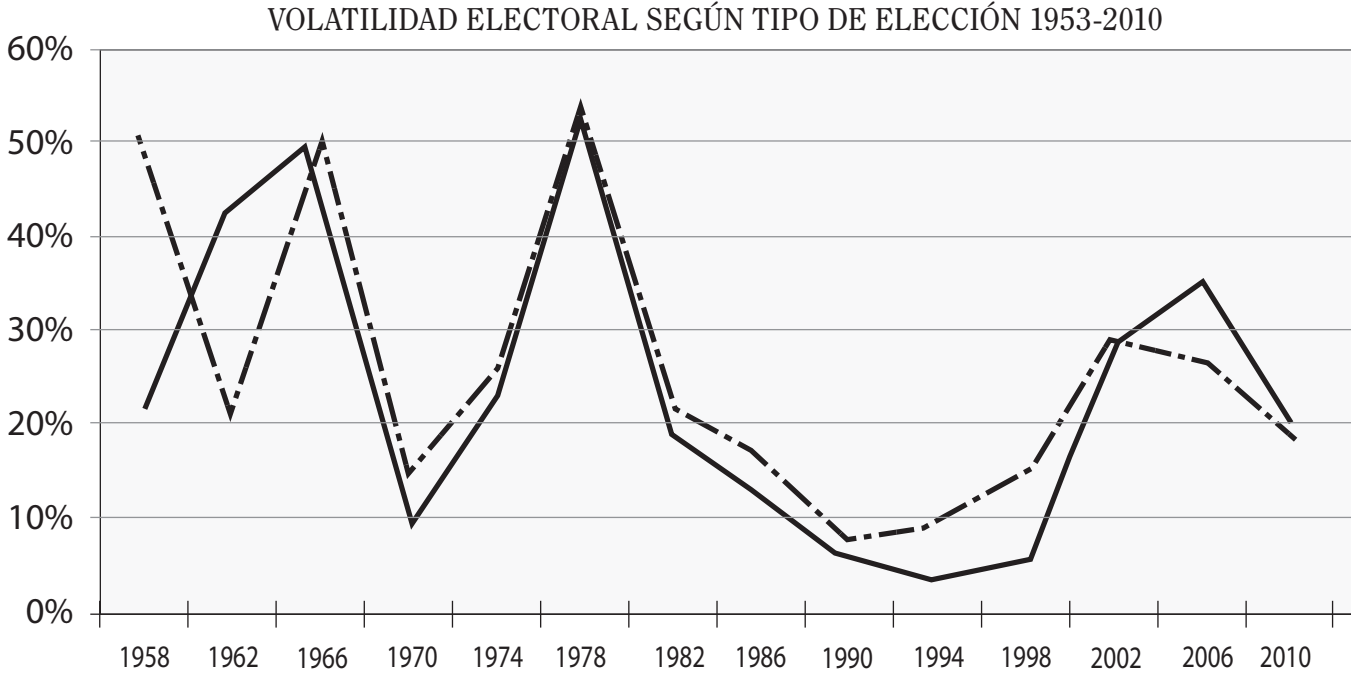

Presidencial _- - L Legislativa

Nota: La volatilidad electoral se calcula mediante el índice de Pedersen, que se obtiene sumando el cambio neto absoluto en el porcentaje de votos para cada partido de una elección a la otra y dividiéndolo entre dos. Fuente: Elaboración propia con datos del TSE. 
En perspectiva comparada los incrementos en la volatilidad electoral en el período 1953-2010, producen alteraciones importantes en la correlación de fuerzas políticas, particularmente en las elecciones de 1966, 1974, 1978, 1998, 2002 y 2006. En estos procesos electorales los cambios en el respaldo generaron desplazamientos y sustituciones de partidos políticos de una elección a otra. Inclusive, algunas agrupaciones desaparecen por completo de la escena política, tal y como sucedió con Unión Nacional en 1962 y Unificación Nacional en 1978. En las fases históricas de mayores niveles de volatilidad se altera significativamente la correlación de fuerzas en las instancias de representación política. Sin embargo, a pesar de que algunas agrupaciones experimentaron incrementos $y$ disminuciones significativas, las transferencias de votos entre partidos de una elección a otra (2006 y 2010) no fueron de la misma magnitud que otras elecciones, particularmente, por el hecho de que el PLN logró retener en 2010 (y desde luego aumentar) buena parte del caudal que había aglutinado en 2006.

Lo interesante del 2010, es que a diferencia de otras elecciones, un cambio en la volatilidad en la preferencia electoral no provoca una alteración en la fragmentación del sistema de partidos. Se esperaría que entre ambos indicadores se diera una relación directa; es decir, que a menor volatilidad menor la fragmentación, pues es menor la cantidad de electores que cambian su preferencia entre una elección $y$ otra $y$ por ende, menos partidos políticos conquistarían escaños. Sin embargo, tal y como se mencionó anteriormente, este no es el caso.

\section{LIGERO REPUNTE EN LA NACIONALIZACIÓN PARTIDARIA}

En la literatura especializada se ha venido empleando un índice para medir la fortaleza relativa de los partidos denominado índice de nacionalización partidaria. Este indicador estima las diferencias territoriales del apoyo electoral. Por nacionalización parti- daria se entiende el grado en que los partidos reciben niveles similares de apoyo electoral en las diferentes regiones del país. La nacionalización partidaria se mide con un índice que se calcula individualmente para cada agrupación política (party nationalization) y también se puede calcular para el sistema de partidos políticos en general (party system nationalization).

Su estimación se basa en el coeficiente de Gini y permite efectuar comparaciones entre partidos, entre sistemas de partidos, entre elecciones y a lo largo del tiempo. La escala del índice es de 0 a 1 . Un nivel de nacionalización partidaria cercano a 0 significa que el partido recibe un $100 \%$ de votos en una provincia y un $0 \%$ de respaldo en el resto. Mientras más se acerque el valor a 1, más "nacionalizado" será el partido o sistema de partidos. En este sentido, dos agrupaciones pueden tener el mismo porcentaje de votos, pero mostrar disparidades importantes en su nivel de nacionalización. Un sistema de partidos "nacionalizado" es aquel en que los niveles de apoyo electoral de la mayor parte de las agrupaciones políticas no varían significativamente entre una provincia $y$ otra. Por el contrario, a mayores diferencias territoriales en el apoyo partidario, menor es el grado de nacionalización (Alfaro, 2006).

El sistema de partidos políticos costarricense muestra en ambas elecciones, presidencial y legislativa, una modesta recuperación en sus niveles de nacionalización. Aun así, la cifra de la elección legislativa es la cuarta más baja del periodo estudiado. A pesar de que no siempre los cambios en los valores de nacionalización partidaria están asociados a la obtención de una mayor o menor proporción de votos de los partidos más votados entre una elección $y$ otra, en esta oportunidad se da una combinación de ambos factores, pues partidos como el PLN y el Movimiento Libertario incrementaron su apoyo electoral al tiempo que el respaldo hacia el PAC disminuyó considerablemente (gráfico 3). No obstante, el repunte de la nacionalización del sistema de partidos es considerablemente mayor en la elección legislativa que en la presidencial. 
ÍNDICE DE NACIONALIZACIÓN PARTIDARIA SEGÚN ELECCIÓN 1953-2006

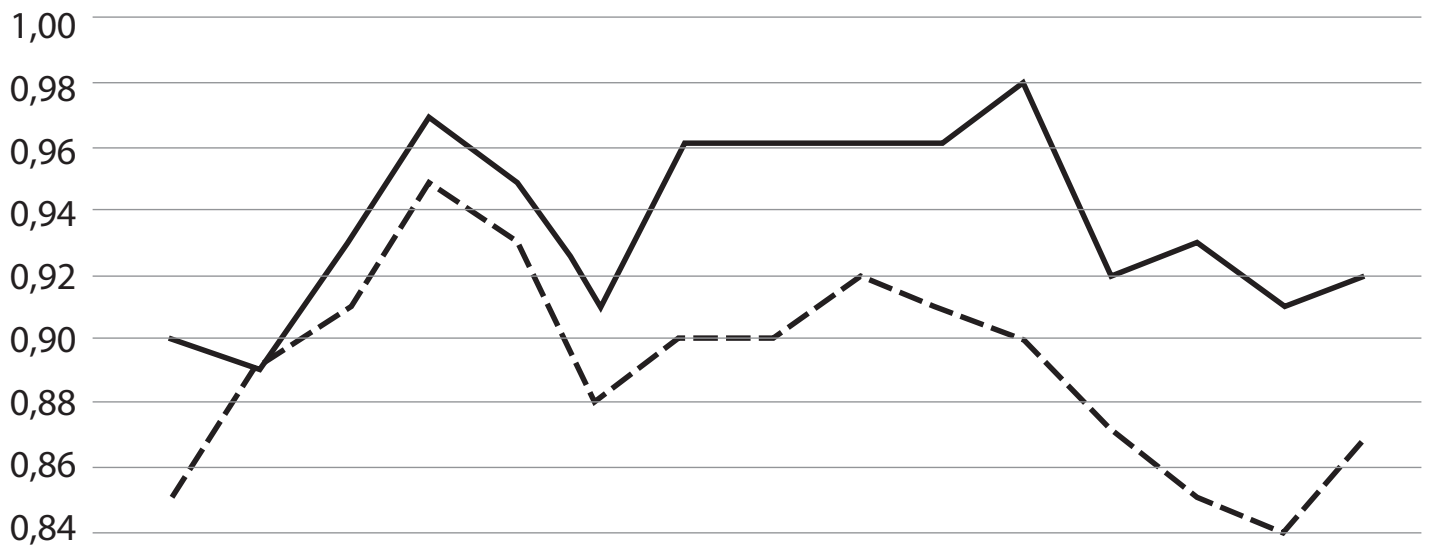

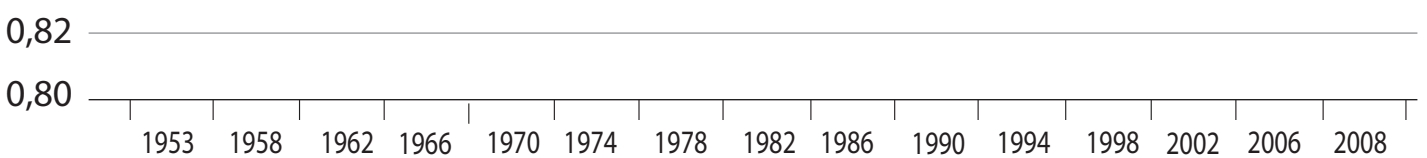

— Presidencial _- Legislativa

Fuente: Elaboración propia con datos del TSE.

Ahora bien, analizados los partidos políticos a modo individual, es factible reconocer diferencias importantes en materia de nacionalización. Previo a las elecciones de 2010, las agrupaciones con mayores niveles de nacionalización eran Liberación Nacional, Coalición Unidad y Unidad Social Cristiana (excepto 2006). Sin embargo con los resultados de los comicios de 2010, queda en evidencia que el partido con la mayor homogeneidad en su apoyo electoral (índice de nacionalización partidaria superior a 0,95) es el PLN, seguido por el Movimiento Libertario y el PAC, cuyos niveles se sitúan alrededor de los 0,9 , en la escala $0-1$. Este resultado evidencia que estas últimas agrupaciones aún se encuentran en un período de consolidación de su apoyo electoral, como lo reflejan sus niveles de nacionalización partidaria que mide el peso de los factores nacionales y locales en el apoyo electoral de los partidos.

Por su parte, en el 2010, la Unidad Social Cristiana fue uno de los partidos que mostró un mayor crecimiento en su nivel de nacionalización, pues creció en la elección presidencial de 0,79 a 0,87 . No obstante, estos valores son considerablemente menores a los que esta misma agrupación obtenía antes de 2006 (Ver gráfico 4).

En síntesis, en lo que respecta al sistema de partidos políticos en el país en la última década, no solo el apoyo electoral de los partidos se ha tornado más frágil y volátil, sino que además los partidos políticos enfrentan mayores obstáculos para conseguir un respaldo electoral homogéneo en el territorio. Los resultados de nacionalización partidaria mencionados constatan la tendencia al debilitamiento de estas agrupaciones a pesar del ligero repunte señalado. Los partidos políticos — nuevos y viejos - deben poner énfasis en la reconstrucción de las relaciones con los diferentes sectores sociales, recuperar la confianza perdida en la ciudadanía, así como, fortalecer sus estructuras internas, particularmente las de reclutamiento, formación partidaria y pensamiento político. 


\section{GRÁFICO 4}

ÍNDICE DE NACIONALIZACIÓN PARTIDARIA EN LA ELECCIÓN PRESIDENCIAL (NO INCLUYE A TODOS LOS PARTIDOS POLÍTICOS)

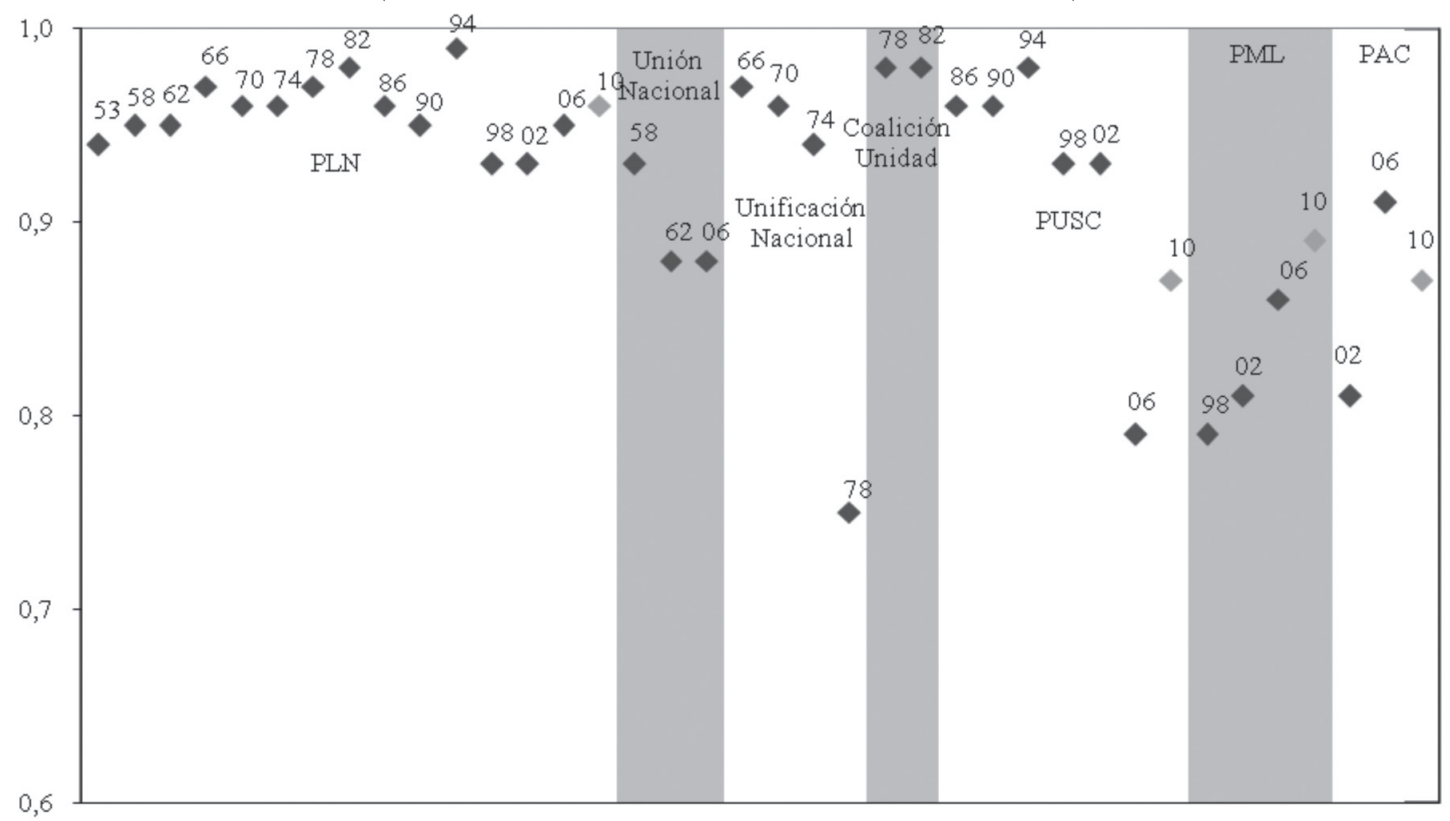

Nota: Cada punto representa un valor de nacionalización según el partido político del que se trate en la elección respectiva. Las cifras que aparecen en la parte superior de cada punto corresponden a las ultimas dos cifras del año en el que se efectuó la elección.

Fuente: Elaboración propia con datos del TSE.

\section{A MANERA DE CONCLUSIONES}

Las elecciones de 2010 no cambiaron radicalmente el panorama incierto en cuanto a la recomposición del sistema de partidos en nuestro país en la época post-bipartidista. Por el contrario, el impase abierto en 2006, luego del desplazamiento del PUSC como segunda fuerza política por el PAC, se extiende en el tiempo y por lo tanto, se reafirma con ello la etapa de transición del sistema de partidos hacia un nuevo e indefinido estadio.

Dos son los factores fundamentales que caracterizan a este periodo inacabado de transición política. Por un lado, una composición pluralista y sin mayorías de los órganos de representación política -Asamblea Legislativa $y$ municipalidades - producto de una mayor fragmentación del sistema de partidos y por el otro, la elevada volatilidad en las preferencias electorales de la ciudadanía. Con respecto a la volatilidad, en 2010 , se produjo una reducción de los cambios netos en la preferencia electoral de los ciudadanos costarricenses, que por las características que muestra podría deberse a aspectos meramente coyunturales.

$\mathrm{El}$ incremento del respaldo electoral del Movimiento Libertario devolvió a los partidos de centro y centro-derecha la predominancia que han ostentado en el escenario políticoideológico costarricense. A pesar de esta reconfiguración, el apoyo electoral a los partidos políticos sigue siendo frágil y volátil. Una vez más la conformación fragmentada de los órganos de representación obligan a los partidos a negociar si lo que pretenden es impulsar su agenda.

Los partidos políticos deben buscar mecanismos para recomponer sus relaciones con los diferentes sectores sociales y particularmente, con una ciudadanía cada vez más apática y desconfiada de sus políticos y apática de cualquier actividad política. Asimismo, es necesario fortalecer sus estructuras internas. 
La nueva legislación electoral introdujo una serie de medidas para renovar el funcionamiento de las agrupaciones políticas que apuntan en la dirección correcta. La democracia costarricense requiere de partidos políticos estables, que se proyecten en las comunidades, que manifiesten y transmitan sus posturas ideológicas $y$ doctrinarias, $y$ aglutinen a importantes sectores de la población más allá de la elección más reciente.

\section{BIBLIOGRAFÍA}

Aguilar Bulgarelli, Oscar R. La forja del Partido Unidad Social Cristiana (su verdadera historia). $1^{\mathrm{a}}$. ed. San José, Costa Rica: Progreso Editorial, 2003.

Alfaro Redondo, Ronald. "Elecciones nacionales 2006 en Costa Rica y la recomposición del sistema de partidos políticos". Revista de Ciencia Política (Santiago) 26. Chile: Pontificia Universidad Católica de Chile, Instituto de Ciencia Política. 2006: 125137. Booth, John A.; Wade, Christine J.; Walker, Thomas W. Understanding Central America: global forces, rebellion, and change. 5th ed. Boulder, co: Westview Press, 2006.

Cerdas, Rodolfo. "The Costa Rican Elections of 1986". Electoral Studies 5 (3). 1986: 311-312.

Feoli, Ludovico. "Costa Rica después del TLC: ¿la calma que sigue a la tempestad?" Revista de Ciencia Política (Santiago) 29. Chile: Pontificia Universidad Católica de Chile, Instituto de Ciencia Política. 2009: 355-379.

Fernández González, Oscar. "Costa Rica: una bipolaridad partidaria hoy apenas cuestionada". Anuario de Estudios Centroamericanos 17 (2). Costa Rica. Editorial de la Universidad de Costa Rica, 1991: 65-74.

Granados Chaverri, Carlos y Ohlsson, Anita. "Organización del territorio y resultados electorales en Costa Rica, 1953-1982". Estudios Sociales Centroamericanos 12 (36). 1983: 183-210.
Hernández Rodríguez, Oscar. "Análisis del abstencionismo en las elecciones presidenciales de Costa Rica en el período 1953-1986". Anuario de Estudios Centroamericanos 16-17 (2-1). Costa Rica: Editorial de la Universidad de Costa Rica, 1990-1991: 117-137.

Laakso, Markku y Taagepera, Rein. "Effective' number of parties: a measure with application to West Europe". Comparative Political Studies 12 (1). 1979: 3-27.

Latin American Public Opinion Project; Universidad de Costa Rica; et ál. "La cultura política de la democracia en Costa Rica: un estudio del Proyecto de Opinión Pública en América Latina. (OPAL)". Americas barometer 2006. Costa Rica. Centro Centroamericano de Población de la Universidad de Costa Rica: USAID, 2005.

Lehoucq, Fabrice Edouard. "The Costa Rican General Elections of 6 February 1994". Electoral Studies 14 (1). 1995: 69-72.

Lehoucq, Fabrice Edouard. "Lucha electoral y sistema político en Costa Rica, 19481998". 1ª . ed. Colección Pasado y Presente. San José, Costa Rica: Editorial Porvenir, 1997.

Lehoucq, Fabrice Edouard; Molina Jiménez, Iván y Ebrary Inc. "Stuffing the ballot box fraud, electoral reform, and democratization in Costa Rica". Cambridge studies in comparative politics. New York: Cambridge University Press, 2002.

Mainwaring, Scott. "Presidentialism, multipartism, and democracy: the difficult combination". Comparative Political Studies 26 (2). 1993: 198-228.

Molina Jiménez, Iván. "Democracia y elecciones en Costa Rica: dos contribuciones polémicas". $1^{\mathrm{a}}$. ed. Cuadernos de Ciencias Sociales. San José, Costa Rica: Facultad Latinoamericana de Ciencias Sociales-Sede Académica. 2001.

Pedersen, Mogens N. "The dynamics of european party systems: changing patterns of electoral volatility". European 
Journal of Political Research 7 (1). March, 1979: 1-26.

Proyecto Estado de la Nación (Costa Rica). Auditoría ciudadana sobre la calidad de la democracia. $1^{\text {a }}$. ed. Pavas, Costa Rica: Proyecto Estado de la Nación. 2001.

Raventós Vorst, Ciska. Abstencionistas en Costa Rica: ¿quiénes son y por qué no votan? $1^{\text {a }}$ ed. San José, Costa Rica: Editorial de la Universidad de Costa Rica-Tribunal Supremo de Elecciones-IIDH/CAPELInstituto de Investigaciones Sociales. 2005.

Rojas Bolaños, Manuel y Sojo, Carlos. El malestar con la política: partidos y élites en Costa Rica. $1^{\mathrm{a}}$. ed. San José, Costa Rica: Flacso, Programa Costa Rica. 1995.

Rojas Bolaños, Manuel; Universidad de Costa Rica; Instituto de Investigaciones Sociales y Departamento Ecuménico de Investigaciones (Costa Rica). "Costa Rica, la democracia inconclusa". Colección Universitaria. San José, Costa Rica: IIS-DEI. 1989.

Rovira Mas, Jorge. Estado y politica económica en Costa Rica, 1948-1970. 2a. ed. San José: Editorial de la Universidad de Costa Rica, 2000.

Rovira Mas, Jorge; Sánchez Machado, Mario A. e Instituto Centroamericano de Documentación e Investigación Social. "La crisis de Costa Rica". Proyecto Crisis y Alternativas en Centroamérica. [Documentos de Trabajo]. San José, Costa Rica: ICADIS. 1986.

Rovira Mas, Jorge; Universidad de Costa Rica e Instituto de Investigaciones Sociales. $L a$ democracia de Costa Rica ante el siglo $X X I .1^{\text {a }}$. ed. San José, Costa Rica: Editorial de la Universidad de Costa Rica. 2001.

Salazar Mora, Orlando y Salazar Mora, Jorge Mario. Los partidos políticos en Costa Rica. $1^{a}$. ed. San José, Costa Rica: Editorial Universidad Estatal a Distancia. 1991.
Salom E, Roberto. "La crisis de la izquierda en Costa Rica". 1'. ed. Colección Debate. San José, Costa Rica: Editorial Porvenir. 1987.

Sánchez C., Fernando F. "Sistema electoral y partidos políticos: incentivos hacia el bipartidismo en Costa Rica". Anuario de Estudios Centroamericanos 27 (1). Costa Rica: Editorial de la Universidad de Costa Rica, 2000: 133-168.

Sánchez C., Fernando F. "Cambios políticos en Centroamérica: el desalineamiento electoral en Costa Rica". Bicentenario Revista de Historia de Chile $y$ América 1 (2). Santiago de Chile: Centro de Estudios Bicentenario, 2002: 117-146.

Sánchez Machado, Mario. Las bases sociales del voto en Costa Rica, 1974-1978. 1'. ed. San José, Costa Rica: Uruk Editores. 1985.

Seligson, Mitchell A. Peasants of Costa Rica and the development of agrarian capitalism. Madison: University of Wisconsin Press, 1980.

Seligson, Mitchell. "Trouble in paradise?: the erosion of system support in Costa Rica, 1978-1999". Latin American Research Review 37 (1). 2002: 160-185.

Seligson, Mitchell A. y Gómez Barrantes, Miguel. "Elecciones ordinarias en tiempos extraordinarios: la economía política del voto en Costa Rica". Anuario de Estudios Centroamericanos 13 (1). Costa Rica: Editorial de la Universidad de Costa Rica, 1987: 5-24.

Urcuyo Fournier, Constantino. Reforma politica y gobernabilidad. San José, Costa Rica: Editorial Juricentro. 2003.

Vargas Cullell, Jorge. "Costa Rica: fin de una era política". Revista de Ciencia Politica (Santiago) 27. Chile. Pontificia Universidad Católica de Chile, Instituto de Ciencia Política, 2007: 113-128.

Vargas Cullell, Jorge. "Costa Rica: una decisión estratégica en tiempos inciertos". Revista de Ciencia Política 
(Santiago) 28. Chile. Pontificia Universidad Católica de Chile, Instituto de Ciencia Política, 2008: 147-169.

Wills-Otero, L. "Electoral Systems in Latin America: explaining the adoption of proportional representation systems during the Twentieth Century". Latin American Politics and Society 51 (3). Fall, 2009: 33-58.

Fecha de ingreso: 07/09/2010 Fecha de aprobación: 25/04/201 
